\title{
MENSURAÇÃO DA GIBOSIDADE E A SUA CORRELAÇÃO COM MEDIDAS RADIOLÓGICAS NA ESCOLIOSE
}

Ângela Kazue Morita, Cláudia Regina Sgobbi de Faria, Célia Aparecida Stellutti Pachioni, Dalva Minonroze Albuquerque Ferreira

Departamento de Fisioterapia da Faculdade de Ciências e Tecnologia - FCT/UNESP. angela.morita@yahoo.com.br

\section{RESUMO}

O tratamento da escoliose necessita de periódicas avaliações radiológicas, aumentando-se os riscos da radiação. O uso de métodos não-invasivos pode ser uma alternativa. O objetivo foi avaliar indivíduos com um instrumento não-invasivo para quantificação da gibosidade e correlacionar estas medidas com os valores dos ângulos de Cobb. Quarenta e um participantes realizaram avaliação antropométrica e teste de Adams, sendo aplicado um instrumento composto por nível d`água e uma régua, para mensurar a gibosidade. Solicitou-se exame radiológico nas medidas $>5 \mathrm{~mm}$. Foi empregado o coeficiente linear de Pearson. A escoliose foi diagnosticada em $68,4 \%$ do total de radiografias. Houve melhor associação entre a medida da gibosidade torácica com o ângulo de Cobb torácico (direito, $r=0,91$; esquerdo, $r=0,83$ ). 0 posicionamento adotado durante o teste de Adams poderia favorecer a melhor correlação dos valores torácicos. Concluímos que o método não-invasivo apresentou melhores resultados para curvas torácicas.

Palavras-chave: escoliose, mensuração da gibosidade, ângulo de Cobb.

\section{MEASUREMENT OF THE HUMP DEFORMITY AND THEIR CORRELATION WITH RADIOLOGICS MEASUREMENTS IN THE SCOLIOSIS}

\begin{abstract}
The treatment of the scoliosis needs periodic radiologics evaluations, increasing the risks of the radiation. The use of methods no-invasivos it can be an alternative. The aim was to evaluate individuals with an instrument noninvasive for quantification of the hump deformity and to correlate these measured with the values of the Cobb angle. Forty one participants accomplished anthropometric evaluation and Adams test, being applied an instrument composed by level plane and a ruler, to measure the hump deformity. It was requested radiologics exam in the measures $>5 \mathrm{~mm}$. The lineal coefficient of Pearson was used. The escoliose was diagnosed in $68,4 \%$ of the total of $x$-rays. There was better association among the measure of the thoracic deformity with the thoracic Cobb angle (right, $r=0,91$; left, $r=0,83$ ). The positioning adopted during Adams test could favor the best correlation of the thoracic values. It was concluded that the method no-invasivo it presented better results for thoracic curves.
\end{abstract}

Keywords: scoliosis, measurement of the hump deformity, Cobb angle. 


\section{INTRODUÇÃO}

A escoliose é uma deformidade complexa que afeta os três planos anatômicos, sendo o desvio lateral no plano frontal, a rotação vertebral no plano axial e a lordose no plano sagital (ASHER; BURTON, 2006; BAGNALL, 2008).

$A$ incidência de escoliose registra valores extremos de 1 até 13\% por todo o mundo. Contudo, os registros clínicos de valores acima de $10^{\circ}$ em programas de avaliação são de cerca de 2\% (FERREIRA; DEFINO, 2001). São poucos os casos em que a sua etiologia é determinada, podendo ser decorrente de processos degenerativos, alterações genéticas ou secundárias a miopatias ou neuropatias. Contudo, aproximadamente $80 \%$ das escolioses é de causa desconhecida, sendo classificadas como escoliose idiopática (ASHER; BURTON, 2006; BAGNALL, 2008).

A relação entre a deformidade da superfície do tronco (gibosidade) e a deformidade vertebral na escoliose não está muito bem documentada do ponto de vista quantitativo, apesar de esta relação ser evidente clinicamente. Diferentes métodos de mensuração são encontrados na literatura (DÖHNERT; TOMASI, 2008; MÍNGUEZ et al., 2007; STOKES; MORELAND; 1989). O uso de um instrumento de madeira, constituído por um nível d’água e uma régua, foi utilizado em vários estudos, fornecendo em milímetros a quantidade de gibosidade presente (FERREIRA; DEFINO, 2001; FERREIRA et al., 2009; 2010; SALATE et al., 2003).

As radiografias têm sido o método mais utilizado para avaliar a evolução do tratamento e o grau de deformidade no diagnóstico. Em decorrência do longo período de tratamento da escoliose, há a necessidade de periódicas avaliações radiológicas, o que tem gerado preocupações quanto aos riscos da radiação. $\mathrm{O}$ uso de métodos não-invasivos para mensurações de escoliose pode ser uma alternativa para acompanhar a sua evolução, reduzindo o uso de radiografias. Ainda, a sua utilização para diagnóstico e acompanhamento de escoliose é mais bem aceito pelo paciente, havendo necessidade de investimento acerca de estudos que visem à utilização desses métodos (FERREIRA; DEFINO, 2001).

\section{OBJETIVO}

Avaliar indivíduos com um instrumento não-invasivo a fim de diagnosticar a escoliose por meio da quantificação da gibosidade clínica e correlacionar estas medidas, em indivíduos com escoliose, com os valores dos ângulos de Cobb encontrados nos exames radiológicos.

\section{METODOLOGIA}

Foram avaliados 41 participantes, 15 do sexo masculino e 26 do sexo feminino, com idades entre 11 e 28 anos. Este procedimento foi composto por avaliação antropométrica e exame cinesiológico.

\section{Exame Cinesiológico}

O indivíduo permaneceu na posição ereta, com o tronco despido e foram então observadas a posição dos membros inferiores, da pelve, da coluna e da cabeça no plano frontal (anterior e posterior) e no plano sagital (direito e esquerdo) e complementou-se com o teste de Adams. Para a realização deste último, o tronco permaneceu descoberto e os participantes assumiram a postura nominal, posicionando-se sobre uma figura da impressão plantar dos pés, previamente estabelecida como forma de padronização. O teste consistiu na realização de uma flexão anterior da coluna vertebral, que pode revelar uma proeminência na superfície do tronco definida como gibosidade. 


\section{Mensuração da Gibosidade}

Para a mensuração da gibosidade foi utilizado um instrumento, que segundo a orientação de Surós (1977), é constituído por um nível d'água de madeira de $35,8 \mathrm{~cm}$ de comprimento, $5,4 \mathrm{~cm}$ de largura e $2,2 \mathrm{~cm}$ de espessura, que é adaptado com um orifício que permite o encaixe de uma régua de madeira de $30 \mathrm{~cm}$, e sobre o mesmo estão fixadas duas réguas de $20 \mathrm{~cm}$ de ambos os lados, para a orientação das mensurações (Figura 1). Para a realização da leitura, em milímetros (mm), considerou-se como referência o nível d'água que se encontrava na posição horizontal (FERREIRA; DEFINO, 2001).

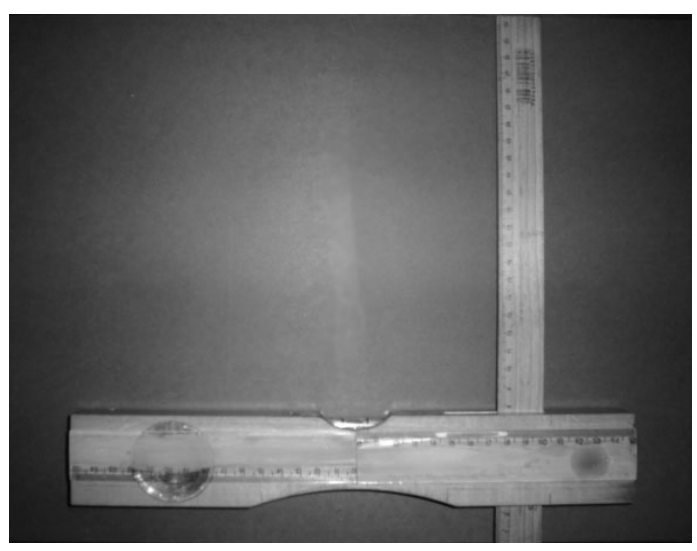

Figura 1. Instrumento para mensurar a gibosidade.

O nível d’água foi apoiado horizontalmente no lado convexo da curva e no ponto mais elevado da gibosidade, mantendo a bolha de ar sempre no centro. Identificava-se a distância da gibosidade até a coluna e em seguida, a régua, encaixada no nível d'água, era apoiada no lado côncavo da curva, usando-se a mesma distância em relação ao lado da gibosidade. Então, registrava-se o valor da maior gibosidade clínica (Figura 2).

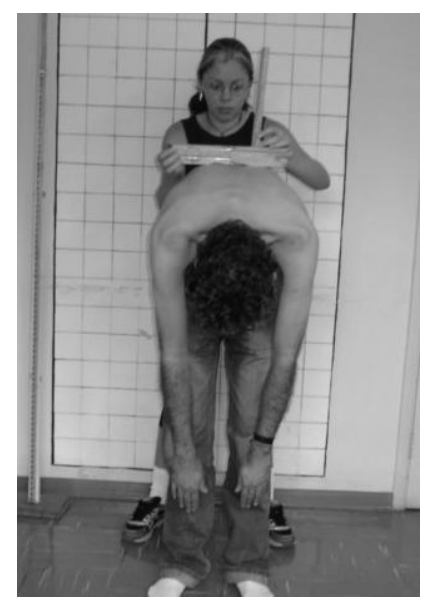

Figura 2. Método de mensuração da gibosidade clínica.

Se apresentassem medidas superiores a $5 \mathrm{~mm}$, os participantes eram orientados a realizarem um exame radiológico para confirmação do diagnóstico de escoliose, detectado nestes casos com ângulo de Cobb maior que $10^{\circ}$.

\section{Análise estatística}

A correlação das medidas de gibosidade com as medidas radiológicas do ângulo de Cobb foi realizada empregando-se o coeficiente linear de Pearson (valor de r). Para tanto, levou-se em consideração a região da coluna (torácica e lombar) e o lado da gibosidade e do ângulo de Cobb (direito e esquerdo).

\section{RESULTADOS}

No presente estudo, dos 41 participantes (peso $=59,1 \pm 12,2 \mathrm{Kg}$; altura $=1,6 \pm 0,1 \mathrm{~m}$; idade $=19 \pm 3,2$ anos), 30 apresentaram alguma alteração da medida da gibosidade (medidas $>5$ $\mathrm{mm})$, sendo $19(63,3 \%)$ do sexo feminino e 11 $(36,6 \%)$ do sexo masculino.

Dentre os participantes que apresentaram alterações na medida da gibosidade, apenas 19 $(63,3 \%)$ realizaram o exame radiológico, sendo 11 mulheres e 8 homens. Foram encontradas 12 curvas torácicas $(38,7 \%), 9$ direitas e 3 esquerdas; 13 curvas lombares $(42,0 \%), 3$ direitas e 10 esquerdas; 6 curvas tóraco-lombares 
$(19,3 \%), 3$ direitas e 3 esquerdas. O diagnóstico de escoliose (ângulo de Cobb $>10^{\circ}$ ) foi confirmado em 13 casos $(68,4 \%)$.

$\mathrm{Na}$ Tabela 1 são apresentados os resultados do teste de correlação, no hemicorpo direito e esquerdo, para o ângulo de Cobb nos níveis torácico e lombar com a medida da gibosidade torácica e lombar. Observa-se que a melhor correlação é dada pela medida da gibosidade torácica com o ângulo de Cobb torácico direito.

Tabela 1. Correlação dos valores do ângulo de Cobb (graus) com a gibosidade (mm).

Gibosidade Torácico
Ângulo de Cobb

\begin{tabular}{lcccc} 
& \multicolumn{2}{c}{ Torácico } & \multicolumn{2}{c}{ Lombar } \\
Torácico & Direito & Esquerdo & Direito & Esquerdo \\
Lombar & 0,91 & 0,83 & $*$ & $*$ \\
& $* *$ & $* *$ & $* * *$ & 0,69
\end{tabular}

* Não é possível correlacionar gibosidade torácica com ângulo de Cobb lombar; ** Não é possível correlacionar gibosidade lombar com ângulo de Cobb torácico; *** Não havia radiografias para correlação.

\section{DISCUSSÃO}

Intercalar medidas não-invasivas entre medidas radiológicas é fundamental para se obter um bom parâmetro quantitativo de acompanhamento das escolioses sem expor os pacientes aos riscos decorrentes da radiação excessiva (FERREIRA; DEFINO, 2001).

As medidas de gibosidade nem sempre apresentam boa correlação com as medidas radiológicas. Isto implica que uma gibosidade mínima de $5 \mathrm{~mm}$ não necessariamente está relacionada com rotação vertebral ou com desvio lateral mínimos, característicos de uma escoliose estrutural (SOUCACOS et al., 1997).

Os valores de correlação encontrados neste estudo estão de acordo com os resultados encontrados em outras pesquisas, nos quais a correlação das medidas da gibosidade com o ângulo de Cobb é melhor para as curvas torácicas que para as curvas lombares (FERREIRA; DEFINO, 2001; PEARSAL et al., 1992). Este fato pode ser decorrente do posicionamento adotado pelo participante para a realização do teste de Adams. Se a inclinação realizada for de $45^{\circ}$, o examinador visualiza o tronco ao nível da coluna torácica, e somente próximo de $90^{\circ}$ que a coluna lombar seria melhor visualizada, sendo que a região tóraco-lombar é observada na transição destes ângulos (BUNNELL; DELAWARE, 1984; FERREIRA; DEFINO, 2001). O melhor resultado para as gibosidades torácicas direitas se deve ao fato de haver maior número de curvas com esta característica.

Ainda, estudos demonstraram que o teste de inclinação anterior tem se mostrado insensível na forma mais comum de escoliose que ocorre na coluna tóraco-lombar. As gibosidades tóracolombares apresentam uma característica não progressiva, ou seja, a medida que a deformidade aumentava, a gibosidade desta região não aumenta na mesma proporção, o que poderia demonstrar um caráter não-linear deste tipo de escoliose (FERREIRA; DEFINO, 2001).

\section{CONCLUSÃO}

O método não-invasivo utilizado nesta pesquisa para mensurar gibosidade clínica apresentou melhor correlação, da gibosidade com o ângulo de Cobb, para as curvas torácicas. Logo, este instrumento poderia ser adequado para diagnosticar ou acompanhar escolioses 
torácicas, porém não poderia ser o único parâmetro nos casos de escoliose lombares ou tóraco-lombares, devendo ser intercalado com medidas radiológicas.

\section{REFERÊNCIAS}

Asher MA, Burton DC. Adolescent idiopathic scoliosis: natural history and long-term treatment effects. Scoliosis 2006; 1(2): 10p.

Bagnall KM. Using a synthesis of the research literature related to the etiology of adolescent idiopathic scoliosis to provide ideas on future directions for success. Scoliosis 2008; 3(5): 7p.

Bunnell WP, Delaware W. An objective criterion for scoliosis screening. The Journal of Bone and Joint Surgery 1984; 64(9): 1381-1387.

Dohnert MB, Tomasi E. Validade da fotogrametria computadorizada na detecção de escoliose idiopática adolescente. Revista Brasileira de Fisioterapia 2008; 12(4): 290-297.

Ferreira DMA, Defino HLA. Avaliação quantitativa da escoliose idiopática: concordância das mensurações da gibosidade e correlações com medidas radiológicas. Revista Brasileira de Fisioterapia 2001, 5(2): 73-86.

Ferreira DMA, Fernandes CG, Camargo MR, Pachioni CAS, Fregonesi CEPT, Faria CRS. Avaliação da coluna vertebral: relação entre gibosidade e curvas sagitais por método nãoinvasivo. Revista Brasileira de Cineantropometria e Desenvolvimento Humano 2010; 12(4): 282289.

Ferreira DMA, Suguikawa TR, Pachioni CAS, Fregonesi, CEPT, Camargo MR. Rastreamento escolar da escoliose: medida para o diagnóstico precoce. Revista Brasileira Crescimento Desenvolvimento Humano 2009; 19(3); 357-368.

Mínguez MF, Buendía M, Cibrián RM, Salvador R, Laguía M, Martín A, Gomar F. Quantifier variables of the back surface deformity obtained with a noninvasive structured light method: evaluation of their usefulness in idiopathic scoliosis diagnosis. European Spine Journal 2007; 16: 73-82. http://dx.doi.org/10.1007/s00586-006-0079-y

Pearsall DJ, Reid JG, Heidden D. Comparison of three nonintensive methods for measuring scoliosis. Journal of Bone Joint Surgery 1992; 79: 85-90.

Salate ACB, Aroni FC, Ferreira DMA. Estudo da evolução a curto prazo da escoliose por meio de mensurações da gibosidade, radiográficas e da dor em adolescentes e adultos jovens. Revista Brasileira de Fisioterapia 2003; 7(1): 39-44.

Soucacos PN, Soucacos PK, Zacharis K, Beris AE, Xenakis TA. School-screening for scoliosis: a prospective epidemiological study in northwestern and central Greece. The Journal of Bone and Joint Surgery 1997; 79(10): 1498-1503.

Stokes IAF, Moreland MS. Concordance of back surface asymmetry and spine shape in idiopatic scoliosis. Spine 1989; 14(1): 73-78. http://dx.doi.org/10.1097/00007632-

\section{0-00015}

